\title{
El rol del Estado peruano en la gestión de los conflictos sociales
}

\section{RESUMEN}

El presente artículo aborda esencialmente el rol que el Estado peruano ha venido desarrollando en los últimos años en la gestión de los conflictos sociales, partiendo de la premisa de que desempeńa siempre un papel protagónico desde sus tres niveles de gobierno. Este trabajo pretende corroborar la tesis de que el rol de gestor implica necesariamente conformarse como un interlocutor en el diálogo, y que ese papel no ha sido cumplido cabalmente por el Estado peruano, produciendo que la gestión de los conflictos sociales sea deficiente.

Palabras clave: Estado, conflictos sociales, diálogo, gestión de conflictos sociales.

\section{The role of the peruvian state in the management of social conflicts}

\section{ABSTRACT}

The present article addresses essentially the role that the Peruvian State has been developing during the recent years in the management of social conflicts, starting from the statement that it always plays a main role in its three levels of government. This paper aims to corroborate the thesis that the manager role necessarily implies being a spokesperson, and that role has not being fully fulfilled by the Peruvian State, causing the management of social conflicts to be deficient.

KEYwORDs: State, social conflicts, dialogue, social conflicts management. 


\section{Los conflictos sociales como síntomas de un problema más profundo}

E conflicto parece ser inherente al ser humano. La palabra tiene origen latín en conflictus que está formado por el prefijo con (convergencia, unión) y la forma en participio de flictus (fligere) que significa "golpe». Podríamos decir que se trata de un golpe junto o, para ponerlo en palabras más sencillas, un pleito. Este primer acercamiento parece denotar que hablamos de una constante en la historia de la humanidad: la pelea.

En este punto debemos definir — con el fin de diferenciar más adelante- lo que entendemos por violencia. Para ellos nos apoyaremos en lo que la Organización Mundial de la Salud define como violencia:

The intentional use of physical force or power, threatened or actual, against oneself, another person, or against a group or community, that either results in or has a high likelihood of resulting in injury, death, psychological harm, maldevelopment, or deprivation ${ }^{1}$. (World Health Organization WHO, 2019)

Esta definición abarca el elemento intencional, el instrumento usado (fuerza física o poder), el sujeto pasivo (persona o comunidad), la consecuencia (heridas, muerte, daño psicológico, afectación del desarrollo o despojo). Sobre esto cabe agregar que la violencia puede ser, además de física, cultura y estructural (Galtung, 1996, pp. 21-48), simbólica (Bourdieu, 1999, pp. 172-175) y psicológica. Estos aspectos de la violencia se van presentando en diferentes fases del proceso conflictivo de forma acumulativa.

Sin embargo, el conflicto no implica necesariamente la existencia de violencia. Los conflictos sociales son situaciones en las que dos o más actores sociales (que puede o no incluir directamente al Estado) perciben que sus intereses se contraponen, y esta contraposición puede degenerar en violencia (Defensoría del Pueblo, 2012, p. 25). Esta situación, de naturaleza compleja, afecta la gobernabilidad democrática, en la medida en que se pueden ver afectadas las actividades de alguna institución pública (como una municipalidad distrital o provincial) o por un hecho más profundo: se pueden ver afectados los derechos fun- damentales de las personas o comunidades, lo cuales deberían ser resguardados por el Estado.

En el Perú, los conflictos sociales han afectado, de maneras aún no cuantificadas, la gobernabilidad. Esta es una de las razones que explican la intervención de la Defensoría del Pueblo en el tema y la creación de oficinas de atención de conflictos sociales en diferentes organismos del Poder Ejecutivo (en especial en gobiernos regionales).

Un conflicto puede presentar episodios de violencia pero no es necesariamente un ingrediente sine qua non para que exista el conflicto social. En el Perú la Defensoría del Pueblo ha registrado una cantidad importante de conflictos sociales desde que destinó una unidad especial para el monitoreo de los mismos, en el año 2004. Según ella ${ }^{2}$, en el año 2016 se registraron 262 casos a nivel nacional, de los cuales fueron resueltos solo 21. Esta proporción se mantiene desde hace algunos años.

Los conflictos sociales atraviesan diversas fases desde que surgen. Primero, se hacen públicas las demandas o el problema que aqueja a algún o algunos actores sociales. Luego, dependiendo de la atención que reciban por parte del Estado, el conflicto puede devenir en un escalamiento de las manifestaciones públicas (o devenir en violencia) o puede canalizarse a través de mecanismos de resolución y/o transformación, que incluyen el espacio de diálogo, la vía judicial, el arbitraje, etc. En el primer caso, la violencia tuvo como resultado, en 2016, a 19 personas fallecidas y 872 heridas.

La Defensoría del Pueblo inició su labor de monitoreo y gestión de conflictos sociales en el año 2004, a partir de los trágicos eventos ocurridos en Ilave (Puno), en los que el alcalde de dicha localidad fue linchado ante las cámaras de la televisión, acusado de haber cometido actos de corrupción. Durante los siguientes años han reportado 1006 conflictos sociales (hasta agosto de 2016), en cifras que fueron elevándose año a año, llegando a alcanzar un pico de 362 casos en el ańo 2010 y que han venido disminuyendo desde el año 2011 hasta alcanzar los 232 casos en 2018. En cuanto a los efectos de la violencia en los conflictos registrados, entre los ańos 2006 y 2015, la

2 Todas las cifras usadas en esta investigación han sido extraídas de los reportes mensuales de conflictos sociales de la Defensoría del Pueblo (https://www.defensoria.gob.pe/areas_tematicas/paz-social$\mathrm{y}$-prevencion-de-conflictos/), salvo que se indique lo contrario. 
GRÁfico 1

Conflictos sociales registrados por la Defensoría del Pueblo, 2004-2019

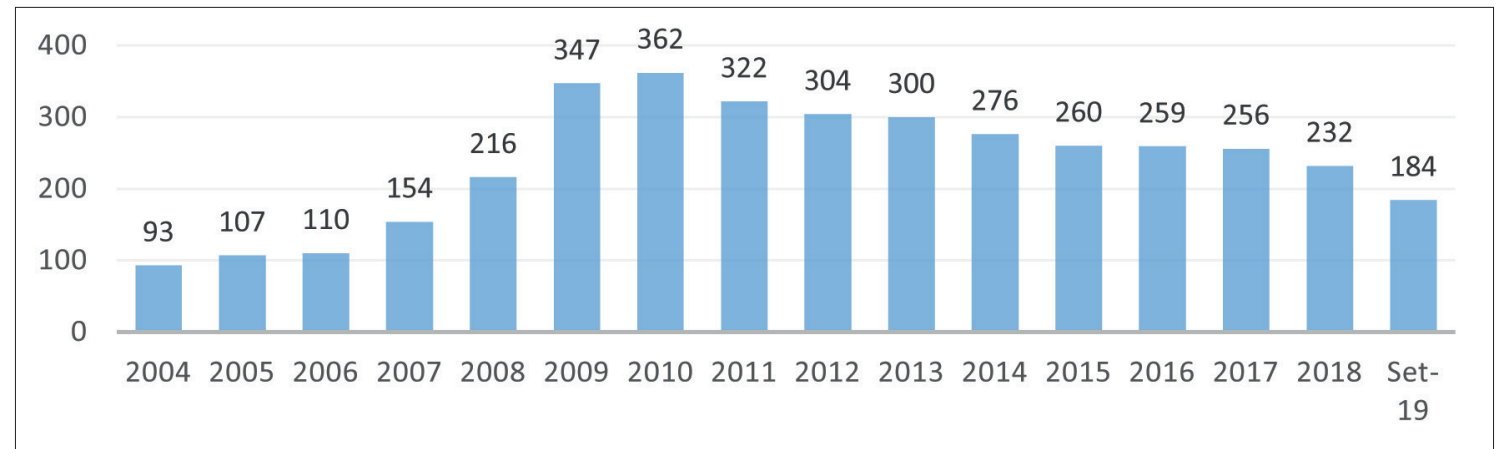

Fuente. Informes anuales de la Defensoría del Pueblo de los ańos 2005 al 2019.

Defensoría registró 264 fallecidos y 4436 heridos, la mayoría población civil.

Al inicio, la mayoría de casos reportados tenían relación con la gestión de las autoridades locales, pero desde el año 2007 los casos socioambientales fueron cobrando protagonismo hasta llegar a representar más del 60\% de los casos que se reportan cada mes. Dentro de este grupo la inmensa mayoría son casos relacionados con la actividad minera. Solamente el año 2016 , estos fueron el $43,5 \%$ de los 260 casos reportados. Los conflictos socioambientales se relacionan principalmente con problemas ambientales y sociales, pero también con el rechazo al inicio de la actividad o el incumplimiento de compromisos.

Cuadro 1

Conflictos socioambientales, según causa aludida, octubre de 2019

\begin{tabular}{|l|c|c|}
\hline \multicolumn{1}{|c|}{ Indicador de agrupamiento } & f & \% \\
\hline Total & 126 & 100,0 \\
\hline Problemas ambientales y sociales & 82 & 65,1 \\
\hline Rechazan el inicio de la actividad & 17 & 13,5 \\
\hline Incumplimientos de compromisos & 27 & 21,4 \\
\hline
\end{tabular}

Fuente. Defensoría del Pueblo. Reporte de mensual de conflictos sociales N. ${ }^{\circ} 188$, octubre de 2019. Elaboración propia.

Otro aspecto relacionado a los conflictos son las protestas sociales, que pueden ser parte de los hechos de un caso de conflicto social o pueden ser manifestaciones aisladas de descontento en un grupo social. La Defensoría ha registrado entre 2008 y 2015 más de ocho mil protestas, la mayoría plantones y marchas que fueron protagonizadas principalmente por trabajadores no sindicalizados y población local. Una de las principales demandas de estas protestas han sido las reivindicaciones laborales (mejora de las condiciones laborales, pago de horas extras, bonificaciones, etc.).

Son diversas las consecuencias de los conflictos sociales, algunas de las cuales llevaron a la Defensoría del Pueblo a intervenir de acuerdo a sus competencias en esta materia (afectaciones a derechos fundamentales, desfavorecimiento de las condiciones para el desarrollo, desestímulo de la cultura del diálogo, etc.). Sin embargo, una de las más terribles consecuencias del estallido de la violencia en los conflictos sociales es el saldo de personas heridas y fallecidas.

Estas cifras muestran que los conflictos sociales en el Perú tienen como consecuencia la vulneración de ciertos derechos y, principalmente, la afectación de la vida, el cuerpo y la salud de las personas que están directamente relacionadas a las protestas pero también de terceros que no se encontraban involucrados en la problemática.

En cierto sentido, la existencia de conflictos sociales puede revelar carencias en cómo se canalizan las demandas sociales o si el rol del Estado es el adecuado. De acuerdo con Remy (Remy, 2004, pp. 18-19), la utilización de espacios no institucionalizados puede significar que otros campos de participación no lograron cumplir con las expectativas de los demandantes o tuvieron costos muy elevados. De esta perspectiva podríamos decir que estamos ante un problema de carencia de canales de participación efectivos o ante un problema de falta de recursos para hacer efectiva la participación. En todo caso, se trata de un problema que afecta la participación ciudadana. 
Gráfico 2. Acciones colectivas de protesta, 2008-2016.

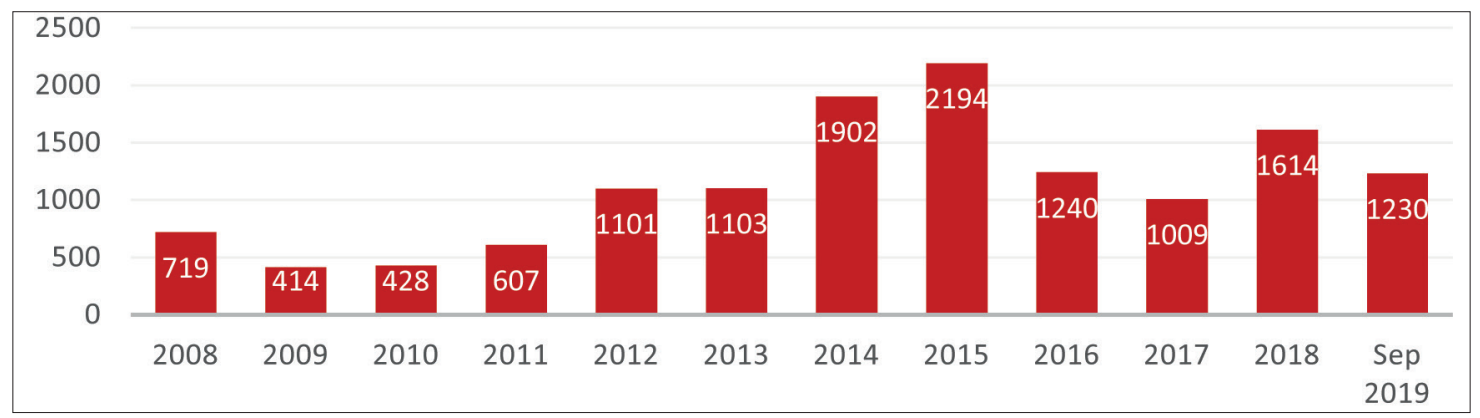

Fuente. Defensoría del Pueblo. Reportes mensuales de conflictos sociales N. ${ }^{\circ} 47$ al 187.

\section{Aproximaciones teóricas a la conflictividad social}

En los últimos quince años se han incrementado las investigaciones sobre conflictos sociales en el Perú. Uno de los primeros trabajos que abordaron la conflictividad en este siglo fue el de José de Echave, "Construyendo un proceso de toma de decisiones frente a operaciones mineras» (De Echave, 2001) que se enfoca en la industria minera, en un momento en el que los conflictos mayoritariamente se daban por demandas hacia las autoridades locales. Unos años después se publica una compilación de trabajos llamada «Los procesos de Diálogo y la Administración de Conflictos en Territorios de Comunidades: El Caso de la Mina de Tintaya en el Perú» (De Echave, Keenan, Romero, \& Tapia, 2005) que también aborda la temática minera en relación con un actor que en adelante sería protagonista: la comunidad campesina.

En 2005 se publica el trabajo «Los múltiples campos de la participación ciudadana en el Perú» de María Isabel Remy, sobre el cual se ha basado una parte importante de esta investigación, que analiza los diversos canales a través de los cuales las ciudadanía puede ejercer el derecho a intervenir en la política pública. Remy plantea que existen cuatro campos sobre los que se desarrolla la participación ciudadana, que se organizan en función a su institucionalidad y la existencia de diálogo con las autoridades (Remy, 2005, p. 16). Así tenemos los mecanismos de democracia directa (institucionalizados pero sin diálogo), las protestas y movimientos sociales (no institucionalizados y sin diálogo), los espacios de concertación (institucionalizados y con diálogo) y la incidencia política (no institucionalizados pero con diálogo).
Hacia el año 2007 el conflicto social ya es un fenómeno social con un gran protagonismo no solo en la academia sino también en la gestión pública pero sin que haya una propuesta articulada de acción, más allá de la que venía trabajando la Defensoría del Pueblo. También aparecen trabajos que ya no solo abordan el conflicto como un tema de investigación sino que van abonando a una forma de gestión. El trabajo de la asociación civil Pro Diálogo es parte fundamental en este sentido, ya que publica trabajos enfocados en la construcción de espacios de diálogo (Caravedo \& Moreno, 2007) y en la construcción de consensos (Bedoya, Caravedo, Moreno, Ormachea, \& Yeomans, 2007). Estas aproximaciones al conflicto desde la gestión son bastante útiles considerando el rol activo que un Estado debe tener hacia este fenómeno social. Entender cómo un gobierno interviene en la conflictividad puede develar constructos teóricos que hay detrás pero además permiten conocer si las fórmulas teóricas resultan tener impacto en la realidad.

En 2009, Tanaka y Grompone publicaron una recopilación de artículos en los que relacionan el conflicto social con el crecimiento económico y la insatisfacción social en el Perú (Grompone \& Tanaka, 2009). Los artículos abordan los conflictos por temas con el gobierno local, las demandas magisteriales, la lucha cocalera por el reconocimiento de la legalidad de su actividad y la disputa casi perenne entre las comunidades campesinas y las grandes empresas mineras en torno al acceso y disfrute de los recursos naturales, y por los efectos ambientales de la actividad extractiva a gran escala. Sobre este último apartado, Arellano exploró los conflictos sociales en zonas de desarrollo de gran minería (Arellano Yanguas, 2014) en las que la distribución de la renta genera actividades productivas no sostenibles, el canon se convierte 
en un incentivo perverso de la participación política $y$ las transferencias fiscales no han logrado traducirse en el mejoramiento de los índices de bienestar.

Eduard Vinyamata (Vinyamata, 2014, pp. 91-136) tiene un acercamiento desde varias disciplinas hacia una definición de lo que es el conflicto. Parte de tomar a la agresividad como un instinto que puede ser necesario para sobrevivir en determinadas circunstancias. La represión de este instinto puede llevar a su estimulación y a desarrollar una mayor virulencia en su manifestación. Por otro lado, yendo a un análisis colectivo de la agresividad, Vinyamata rescata diversas posiciones de autores como Marx, Marcuse, Fanon y otros, que plantean que la sociedad es la generadora de los conflictos y, por lo tanto, la que estimula la agresividad.

Existen también acercamientos desde la psicología que plantean que existe un carácter sociable innato en las personas que se va perdiendo debido a los miedos. Otros psicólogos le atribuyen a la agresividad un origen cultural provocado por frustraciones originadas en la infancia. Los planteamientos desde la antropología de Malinowski y Margared Mead señalan que la agresividad forma parte de los valores colectivos de ciertos grupos humanos, mientras que los sociólogos Parsons y Smelser consideraban a los conflictos como aberraciones que perjudicaban la cohesión de las sociedades.

El conflicto ha sido abordado desde la Sociología desde hace bastante tiempo aunque no con la especificidad que surgió desde los ańos sesenta en adelante. Consideraremos al marxismo como la primera escuela que brinda una aproximación teórica. Luego se haría lo propio desde el funcionalismo. En adelante hay un grupo de teorías que ya pueden llamarse teorías del conflicto.

\section{El enfoque marxista}

El Manifiesto del Partido Comunista pone en su primera parte que "La historia de toda sociedad hasta nuestros días no ha sido sino la historia de las luchas de clases» (Marx \& Engels, 2011, p. 30). Parte de los fundamentos de la teoría marxista tiene que ver con concebir a la sociedad como un cuerpo organizado en partes llamadas clases sociales, las que se diferencian dependiendo su relación con los medios de producción. La posición que detentan surge de esta relación, y esto también conlleva a que sus intereses sean diferentes y, sobre todo, antagónicos. Mientras que los burgueses son los dueños de los medios de producción, los proletarios solo son dueños de su fuerza de trabajo. El valor generado por la fuerza de trabajo de los trabajadores genera la plusvalía de la cual se apropia el burgués. Ambas clases coexisten en constante contradicción

Esta es parte fundamental de la teoría marxista, ya que plantea que mientras existan clases, existirá conflicto entre ellas y este es el motor del proceso social, que, como sabemos, es teleológico en Marx. Las clases son los sectores en los que está dividida la sociedad, de acuerdo con su relación con los medios de producción. Principalmente, Marx y Engels se refieren a dos clases: proletarios y burgueses. Los intereses de ambas clases son distintos en cuanto una tiene la propiedad de los medios de producción y la otra no. A esto le añadieron otro elemento fundamental que es la conciencia de clase o, dicho de otro modo, el autorreconocimiento como clase social que va acompañado de un proyecto político. Los elementos recién descritos permiten entender el surgimiento de los conflictos dentro de la sociedad que van a decantar en lucha de clases.

Los movimientos sociales no fueron plenamente abordados por Marx y Engels pero sí por Lenin, quien incorporó el concepto de vanguardia revolucionaria (Lenin, 1974, pp. 108-128), que vendría a ser la élite dirigente del movimiento revolucionario y que tiene el rol de organizar a las masas a través del partido. Por otro lado, Gramsci propuso que el eje central revolucionario no estaba en la estructura económica y la organización, sino que estaba en la interacción política institucional y la cultura (Gruppi, 1978, pp. 7-24, 89-111). Más recientemente, E.P. Thompson y Eric Hobsbawn plantearon, a partir de sus estudios sobre la clase obrera británica, que la movilización obrera surge de la identidad colectiva, formada por elementos ajenos al movimiento en sí (como las tradiciones compartidas).

\section{El enfoque estructural-funcionalista}

Otro gran enfoque de los conflictos desde la Sociología ha sido el funcionalismo. La sociedad, desde este cuerpo teórico, está conformada por instituciones que sirven para satisfacer alguna necesidad 
social en particular, es decir, desempeñan una función. Los funcionalistas, en general, reconocen algunos elementos comunes como son el sistema social (actores que interactúan en busca de ganancias utilitarias), la estructura (el estado, las normas, los valores, etc.) y la función (basada en tres paradigmas relativos a la acción social).

Los teóricos funcionalistas tuvieron un enfoque bastante conservador sobre la sociedad. De sus postulados desprendieron la idea de que la armonía es el elemento cohesionador y común en la vida social; que todo elemento en la vida social (material o inmaterial) desempeña un papel indispensable en la sociedad; y que los actores comparten un conjunto de valores y fines. En este esquema el conflicto social es tomado como una desviación que atenta contra la sociedad y que, por lo tanto, debe ser suprimido.

Merton tiene un enfoque más laxo sobre el conflicto social. Él incorporó a la propuesta funcionalista el concepto de función latente, que no es manifiesta ni es conocida por los actores sociales pero que contribuye al orden social. Dentro de estas encajó al conflicto como una disfunción, es decir, una alteración de la armonía social pero que funcionaba como válvula de escape y permitía mantener todo en equilibrio.

\section{Enfoques neomarxistas}

Más recientemente, Coser — también desde el cristal estructuralista - definió al conflicto social como la lucha por los valores y por el estatus, el poder y los recursos escasos, en el curso de la cual los oponentes desean neutralizar, dañar o eliminar a sus rivales

Además, le atribuyó funciones al conflicto social que lo ubican como un mecanismo de válvula de escape de la hostilidad que genera el disentimiento entre los miembros de la sociedad (Coser, 1961, pp. 62-68) . El conflicto tiene como una de sus funciones la de establecer y reafirmar la identidad del grupo, que es una forma de mantener las divisiones sociales con base en el antagonismo.

Por otro lado, Coser diferencia entre conflictos reales e irreales, siendo estos últimos aquellos cuya disputa no se centra en algún recurso existente en la realidad sino en la necesidad de liberar la tensión generada por la disputa del recurso. Este punto es importante para entender la aparición de los impul- sos hostiles, que surgen de la acción recíproca sujetoobjeto. El conflicto real no siempre involucra una tensión a nivel psicológico o emotivo, pero un actor puede llevarlo a este plano con el fin de fortalecer la disposición de los demás actores de llevar el conflicto hasta las últimas consecuencias.

El antagonismo de los actores, dice Coser, suele ser más intenso en los conflictos que involucran relaciones sociales íntimas, debido a la fuerte acción recíproca que caracteriza a este tipo de relaciones. El apasionamiento es mayor y, por lo tanto, el conflicto tiende a ser más radical (hacia la unión o la oposición). En grupos cerrados, el sentimiento de amenaza a la cohesión del grupo es un ingrediente para la intensificación del conflicto.

Al enfocar los sentimientos hostiles hacia sucedáneos, se evita el cuestionamiento total del sistema. Asimismo, Coser le atribuye al conflicto social la capacidad de moldear las relaciones y fortalecerlas al generar consensos sobre los temas en controversia (siempre y cuando no se trate de elementos fundamentales del grupo) e incentivar la formación de alianzas y coaliciones que mantienen el equilibrio de poderes.

A mediados del siglo xx, Ralf Dahrendorf aportó una perspectiva sobre el conflicto social que hizo dialogar los enfoques marxista y estructural-funcionalista. Partiendo de preguntarse qué es lo que mantiene a una sociedad unida, llegó a conclusiones que se sostienen en la estructura del conflicto y la naturaleza del cambio social. La sociedad, para Dahrendorf, necesita de cierta estabilidad, pero el conflicto siempre estará, adoptando formas violentas o pacíficas.

Los conflictos sociales, para este autor, no son casuales sino que son parte de la sistemática de la estructura de la misma sociedad. En ese sentido, el orden solo puede existir dentro de una evolución que está marcada por el compás de las pugnas y oposiciones. Con esta consideración — de que no hay sociedad sin conflicto social- Dahrendorf plantea una teoría sobre el conflicto social que es capaz de responder a las siguientes preguntas:

1. ¿Qué hay que entender por conflicto social y qué clase de conflictos podemos distinguir en las sociedades históricas?

2. ¿Dentro de qué imagen social se ofrecen los conflictos? 
3. ¿Cómo se pueden determinar los puntos de partida estructurales de los conflictos sociales?

4. ¿De qué modo se despliegan las situaciones conflictivas en sus relaciones internas?

5. ¿Cuáles son las dimensiones de la variabilidad de cada conflicto?

6. ¿De qué modo pueden regularse los conflictos sociales?

(Baquer, 2000, p. 159)

Finalmente, Dahrendorf incorpora al análisis algunas categorías que contribuyen a entender el fenómeno estudiado. Violencia e intensidad son dos de ellas que, si bien pueden aparentar similitud, aluden a aspectos diferenciados. La intensidad mide la cantidad de actores que participan del conflicto, mientras que la violencia puede ser ejercida por un grupo reducido de actores sociales.

\section{Nuevas teorías del conflicto social}

A partir de la década de los sesenta, surgen nuevos enfoques sobre el conflicto social desde otras disciplinas (ciencia política, antropología, historia, psicología). Dos de estos enfoques principales son las propuestas de Mancur Olson (teoría de la elección racional) y Charles Tilly (teoría de la movilización de recursos y de las oportunidades políticas).

Olson explica el movimiento social a partir de la elección racional costo/beneficio del individuo (Olson, 1985, pp. 32-55), basada en las expectativas que tiene (económicas) y en el riesgo que está dispuesto a asumir. Esto significa que el individuo participará en el movimiento social en tanto el beneficio esperado de participar sea superior a la posible pérdida (riesgo) que podría tener. Esto supone, sin embargo, que existe únicamente una motivación individual de los sujetos para sumarse al colectivo. ¿Quiénes entonces pueden mantener causas altruistas sobre una suma de individualidades que buscan maximizar su beneficio y reducir su riesgo?

Esta sería tarea de una vanguardia, similar a la que desde el marxismo planteó Lenin pero nuevamente surge la pregunta ¿cómo movilizar individuos hacia una causa altruista? El mecanismo que plantea Olson es la figura del incentivo selectivo. Esto es una forma de maximizar el beneficio de quienes son partícipes del movimiento (como por ejemplo, el otor- gamiento de mejores remuneraciones para los miembros activos de un sindicato movilizado). El elemento económico no es lo único que explica el surgimiento del movimiento social. Charles Tilly añadió en los años setenta el concepto de oportunidades políticas (Tilly, 1978, pp. 109-111), que plantea que la fortaleza o debilidad de las autoridades o élites dirigentes configura un escenario más o menos favorable para el surgimiento del movimiento. Desde esta perspectiva, la lucha por el poder es un elemento central en la vida del conflicto social.

Otras propuestas interesantes surgen a partir de los años 80, que pretenden explicar el escenario en el que surge el movimiento social. Estos enfoques vinculan el conflicto con procesos político-culturales y con escenarios favorables para el surgimiento del conflicto (redes, tradiciones, identidades, etc.). Sin embargo, estas formulaciones resultan muy enfocadas en la experiencia europea y estadounidense del siglo xx, y dejan de lado elementos importantes de la cultura y la ideología.

Ya entrando en el siglo XXI, identificamos a tres autores que presentaron aproximaciones nuevas al análisis del conflicto social. Lorenzo Cadarso aporta un enfoque histórico que amplía el análisis tanto de historiadores como de conflictólogos (Lorenzo Cadarso, 1995, pp. 244-245). Para Remo Entelman, desde una aproximación jurídica, la teoría de conflictos debe encargarse de describir el enfrentamiento en sí, analizar sus elementos y formas de presentarse (Entelman, 2002, pp. 53-54). Esta propuesta, si bien nos da una lectura que enriquece el debate, no recoge la teoría ya existente.

Desde la psicología social, Dean Pruitt y Sung Hee Kim aportaron una herramienta de análisis a nivel micro que no se había tocado antes: el análisis de la percepción recíproca de los actores (Pruitt \& Kim, 2004, pp. 39-59). Cuando la estimación de un actor sobre sí mismo es alta, y sobre su antagonista es negativa, irremediablemente habrá contienda. Cuando no confía en sus propias capacidades frente el otro actor, el resultado será la inacción o la retirada. Cuando ambas partes confían mucho en sus capacidades, lo que resultará es la negociación. Finalmente, cuando hay poca estima en el potencial de sí mismo y hay temor del adversario, lo que se dará es una concesión. 


\section{¿El conflicto es positivo o negativo?}

Luego de explorar los diversos abordajes del conflicto social, desde las principales corrientes del pensamiento sociológico ( $\mathrm{y}$ algunos aportes desde otras disciplinas), podemos afirmar que hay dos posiciones directamente en contradicción respecto a la valoración que tiene el conflicto social para la sociedad.

Las definiciones estructuralista y estructural-funcionalista del conflicto social lo incorporan como un elemento que no es intrínseco a las relaciones sociales, y que debe ser suprimido en vista de que pone en peligro las bases sobre las cuales se construyen. El conflicto viene a ser la expresión macrosociológica de la conducta anómica del individuo. Por tanto, la existencia del conflicto en la sociedad no solamente no aporta a la cohesión sino que la destruye.

La posición marxista, si bien tiene al conflicto como un producto esperado de las relaciones de clase, también coincide en que su efecto puede resultar destructivo pero también creador, ya que el resultado de la lucha de clases no da lugar a la nada sino que crea nuevas relaciones entre los grandes grupos sociales. En la dialéctica del conflicto social reside su poder creador de nuevas superestructuras sociales. Por otro lado, los nuevos enfoques (incluyendo los neomarxistas) atribuyen funciones muy concretas y específicas al conflicto social. Lo asumen también como un componente congénito de la sociedad. La cohesión social, desde estas perspectivas, se ve fortalecida por las relaciones generadas en la dinámica del conflicto.

\section{¿Por qué surgen los conflictos sociales?}

Siguiendo nuestra definición de conflicto social podemos decir que estos surgen porque la sociedad existe. Hemos dicho que en toda sociedad existen intereses diversos y que pueden ser contradictorios. En democracia los conflictos se hacen visibles más rápidamente, mientras que en regímenes autoritarios estos se hacen subrepticios y se manifiestan públicamente de forma muy violenta, llegando a la rebelión o la guerra civil.

Las sociedades no son conjuntos homogéneos de grupos humanos. Inclusive las sociedades que aparentan ser homogéneas, es decir, que comparten un mismo patrón cultural (idioma, costumbres, re- ligión, etc.) y hasta un mismo grupo étnico, tienen matices dentro que pueden ser menos pronunciados que sociedades pluriculturales. La heterogeneidad de las sociedades radica en su composición, puesto que toda sociedad se encuentra, de alguna u otra forma, estratificada. Las divisiones por estamento, casta o clases social, determinan en gran medida el carácter conflictivo de las sociedades. Existe la diversidad y oposición de intereses, y por lo tanto existe la disputa. La forma cómo se puede canalizar la contraposición y la forma cómo se desempeñan los actores sociales puede determinar si una situación se torna en conflicto social o no.

En los países industrializados, los conflictos sociales más importantes han sido por temas laborales y por políticas económicas. Esto lo hemos visto en grandes movilizaciones sociales en Noruega ${ }^{3}$ en el ańo 2000, convocadas por las centrales sindicales que exigían mejores condiciones salariales, y que movilizaron a más de 82000 trabajadores. En Suecia ${ }^{4}$, en 2013, se movilizaron trabajadores, la mayoría jóvenes y migrantes, contra las políticas de empleo que no favorecían a estos sectores. En Francia, durante el año en curso, se dieron grandes movilizaciones violentas contra las políticas económicas para afrontar la crisis, situación que vivió España con las movilizaciones de «indignados» en 2011.

En los Estados Unidos, los conflictos sociales han tenido motivaciones menos ligadas a lo económico. Las últimas protestas masivas han tenido origen en los casos de violencia policial contra la población afroamericana, que han sido muchos en los últimos años (o que al menos han tenido mayor difusión en los medios de comunicación tradicionales). Otro caso particular es el de Islandia, en el que una enorme movilización produjo la renuncia del primer ministro por estar ligado al caso de corrupción denominado Panama Papers.

En la protesta en general, la tecnología de las comunicaciones ha tenido bastante importancia en los últimos años. Las protestas que dieron lugar al derrocamiento del régimen de Ben Ali en Túnez, durante la llamada «Primavera Árabe», se transmitían y con-

3 http://elpais.com/diario/2000/05/04/economia/957391224_ 850215.html

4 http://www.abc.com.py/edicion-impresa/internacionales/desempleo-y-crisis-alientan-conflictos-sociales-en-suecia-577446.html 
vocaban usando las redes sociales. ${ }^{5}$ Esto tenía mucho sentido en países en los que no existía pluralidad en los intereses defendidos por los grandes medios de comunicación masiva. Esto mismo se replicó en Argelia, Mauritania, Omán, Yemen, Siria, Egipto, etc.

En el Perú, los conflictos surgen porque la población de una comunidad exige a una empresa minera que cumpla con compromisos pactados o porque se oponen a la actividad hidrocarburífera por considerarla peligrosa. Surgen porque un sindicato no ve atendido su pliego de reclamos ante su patronal o porque los comerciantes de un mercado se niegan a adecuarse a las nuevas disposiciones municipales por considerarlas perjudiciales a su actividad empresarial. Estamos ante situaciones más diversas que lo ocurrido en los países industrializados. Contamos razones ambientales, de gobierno local, laborales, de demarcación territorial, etc.

En la región, la gran mayoría de conflictos sociales tienen origen en situaciones muy similares. En Bolivia ${ }^{6}$, los principales conflictos sociales tienen causas ambientales, de demarcación territorial y por temas agrarios. En Ecuador ${ }^{7}$, los conflictos son por temas ambientales, específicamente por la Ley de Minería aprobada durante la segunda gestión de Rafael Correa. En Chile ${ }^{8}$, los conflictos son básicamente ambientales aunque los más visibles en medios internacionales han sido los ligados a las protestas estudiantiles por una educación gratuita. Estos tres países viven, en estos momentos, convulsiones sociales importantes por temas ligados a la institucionalidad democrática (Bolivia) y el modelo económico (Ecuador y Chile).

Como se puede notar, los conflictos sociales van muy ligados al momento político-económico que atraviesa un país. En los países industrializados existen también disputas sobre temas ambientales pero no llegan al conflicto social como sí lo hacen las movilizaciones en contra de políticas de recorte de empleos. En nuestra región (y en nuestro país), los proyectos mineros o hidroeléctricos parecen venir con un conflicto bajo el brazo.

5 La inmolación de Mohamed Bouazizi frente al Palacio de Gobierno fue transmitida por redes sociales y fue, para muchos entendidos del tema, el desencadenante de las protestas en todo el país.

6 http://www.alterinfos.org/spip.php?article6857

7 http://www.cetri.be/Conflicto-social-y-cambio-politico?lang=fr https://nacla.org/article/correa-vs-movimientos-sociales-conflictoen-ecuador

8 https://www.bpdigital.cl/opac/...de_conflictos...Chile.../libro_web_ descargable.pdf
Existen también razones que van por el lado ideológico. Un ejemplo claro fue el conflicto generado a raíz de la inclusión del enfoque de igualdad de género en la currícula de educación básica regular, por parte del Ministerio de Educación como parte de sus funciones como ente rector en el sector. Este enfoque generó el rechazo de sectores ultraconservadores de la sociedad (encabezados por líderes de iglesias evangélicas, algunos de ellos con escańos en el congreso).

La ideología es una herramienta para aprehender al medio social, político y económico. Los casos por temas laborales tienen, inherentemente, un conflicto basado en contraposición ideológica respecto a la participación de los trabajadores en el aprovechamiento de la plusvalía generada. Los casos por temas mineros o hidrocarburíferos (y en general donde hay grandes proyectos) tienen también una tensión ideológica respecto a la propiedad de la tierra, el uso, los medios de producción y la visión de desarrollo de las partes involucradas.

Esto nos lleva a entender también las posiciones políticas de los actores, las cuales están ciertamente marcadas por sus posiciones ideológicas. El Perú inició el proceso de descentralización durante el gobierno de Toledo (2001-2006) y, como mencionamos en la introducción, se generó una gran expectativa en la población respecto a lo que las autoridades locales podían lograr. Estos nuevos poderes locales terminaron de desmembrar lo poco que quedaba de partidos nacionales.

Las nuevas fuerzas políticas regionales (organizaciones políticas de origen y con demandas locales y/o regionales que fueron ganándoles las elecciones a los tradicionales partidos nacionales) se fueron consolidando, con agendas muy claras para sus regiones y localidades. Empero, en los lugares donde los conflictos por grandes proyectos surgieron, apareció una exigencia de la población y organizaciones de la sociedad civil: que las autoridades locales representen los «intereses del pueblo» y no del gobierno central. Las primeras respuestas de la autoridades locales no fue la de tomar parte, y eso les costó el cargo en la siguiente elección. Aquellos líderes que encabezaron las protestas se erigieron como nuevas autoridades y cambiaron el papel de los gobiernos subnacionales al tomar posición en favor de la población.

El caso más saltante es el de Tía María, en el que los alcaldes distritales y el provincial se sientan a la mesa con ministros y otros funcionarios del Ejecutivo 
pero no para coordinar acciones sino para negociar la salida al conflicto. Los gobiernos de Toledo, García, Humala y PPK no tuvieron cómo hacer frente a este fenómeno porque sus partidos no tenían bases regionales ni locales, eran un collage de candidatos de distintas ramas políticas.

\section{Espacios de participación ciudadana}

En el Perú existen desde hace algunos años, diversos mecanismos de participación ciudadana a través de los cuales la sociedad puede canalizar demandas y pedir solución a problemas que les afectan. Sin embargo, estos mecanismos demandan diferentes costos a los ciudadanos para poder ser utilizados, lo que conlleva a que, a pesar de su existencia, algunos no puedan ser utilizados. En esa misma lógica, existen mecanismos que son los más utilizados y que están más relacionados con la protesta social.

La Constitución Política del Perú reconoce el derecho a participar en la vida política del país: «Toda persona tiene derecho: [...] A participar, en forma individual o asociada, en la vida política, económica, social y cultural de la Nación. Los ciudadanos tienen, conforme a ley, los derechos de elección, de remoción o revocación de autoridades, de iniciativa legislativa y de referéndum». ${ }^{9}$ Asimismo, la Ley Marco de Modernización del Estado ${ }^{10}$ aborda la democracia participativa asignando al Estado el deber de "promover y establecer los mecanismos para lograr una adecuada democracia participativa de los ciudadanos, a través de mecanismos directos e indirectos de participación", y al ciudadano el derecho a "participar en los procesos de formulación presupuestal, fiscalización, ejecución y control de la gestión del Estado, mediante los mecanismos que la normatividad establezca».

Estos mecanismos se van plasmando, por ejemplo, en la Ley de Procedimiento Administrativo ${ }^{11}$, que otorga a los ciudadanos el derecho a "participar responsable y progresivamente en la prestación y control de los servicios públicos, asegurando su eficiencia y oportunidad.»

Para María Isabel Remy, una forma de entrar a analizar el campo de la participación ciudadana es

9 Constitución Política del Perú.

10 Ley N. ${ }^{\circ} 27658$.

11 Ley N. 27444. utilizando algunos criterios que ordenan todo el panorama de mecanismos existentes. El primer criterio sería la verificación de si el mecanismo es o no institucionalizado, es decir, formalizado a través de normas legales. El otro criterio es si el mecanismo tiene por objetivo el diálogo con las autoridades o si se busca obligar a una autoridad de tomar una determinada decisión. A esto le hemos añadido un campo para congregar todos aquellos espacios creados a partir de la gestión de conflictos y que tienen por fin atender demandas o crear condiciones de desarrollo que contribuyan a resolver los problemas que generaron el conflicto. Tenemos:

Cuadro 2

Criterios para analizar los mecanismos de participación

\begin{tabular}{|c|c|c|}
\hline & Institucionalizados & $\begin{array}{c}\text { No } \\
\text { institucionalizados }\end{array}$ \\
\hline $\begin{array}{l}\text { Imposición } \\
\text { de la } \\
\text { voluntad } \\
\text { ciudadana }\end{array}$ & $\begin{array}{c}\text { I } \\
\text { Mecanismos de } \\
\text { participación directa. }\end{array}$ & $\begin{array}{c}\text { II } \\
\text { Protestas y } \\
\text { movimientos } \\
\text { sociales }\end{array}$ \\
\hline $\begin{array}{l}\text { Diálogo con } \\
\text { autoridades } \\
\text { (propuestas) }\end{array}$ & $\begin{array}{c}\text { III } \\
\text { Consejos de } \\
\text { concertación; mesas de } \\
\text { lucha contra la pobreza, } \\
\text { CCL }\end{array}$ & $\begin{array}{c}\text { IV } \\
\text { Propuestas, } \\
\text { incidencia }\end{array}$ \\
\hline $\begin{array}{l}\text { Espacios } \\
\text { ad-hoc }\end{array}$ & $\begin{array}{c}\mathrm{V} \\
\text { Comisiones, comités, } \\
\text { grupos de trabajo, etc. } \\
\text { creados para atender } \\
\text { conflictos y/o generar } \\
\text { condiciones para el } \\
\text { desarrollo. }\end{array}$ & \\
\hline
\end{tabular}

Fuente. Remy, 2004, p. 16.

En el primer campo se encuentran los mecanismos que a través de los cuales la ciudadanía puede cambiar el marco legal que el Congreso aprueba o cambiar a autoridades locales y regionales electas. En estos mecanismos, cuando se cumple con todos los requisitos, se ejecutan las iniciativas, no se negocian. Es también el que demanda mayores costos en el sentido de organización pero también en un sentido más amplio que incluye logística organizativa. Suelen llevarse a cabo, en el Perú, como revocatorias a autoridades distritales y, como una evento más reciente y aislado, un revocatoria a niveles metropolitanos (revocatoria a Susana Villarán). Estos mecanismos son los que mayormente se asocian con la participación ciudadana. 
En el campo III se desarrolla lo que más comúnmente se suele relacionar con la participación ciudadana. Es un campo donde se puede llegar a verdaderos cambios institucionales (como los que se dieron durante el gobierno de Paniagua). Los requisitos para participar son distintos a los del primer campo y son básicamente la pertenencia a organizaciones de la sociedad civil. Tiene la característica de que quienes convocan a estos espacios definen a los participantes y los temas a tratar, además que ser un campo de participación personalista. Es un campo con menos costos pero también menos eficacia.

El campo IV es el espacio del cabildeo y de presentación de iniciativas por parte de organizaciones, ONG o plataformas, que demandan costos muy altos (generalmente financiados por la cooperación internacional). La presentación de iniciativas en este campo demanda equipos técnicos o especializados.

El campo II es el que Remy llama el de la "participación conflictiva» no institucionalizada. Se trata básicamente de las acciones de fuerza que la población toma para obligar a una autoridad a atender una agenda. Este campo, señala la autora, implica que los otros mecanismos de participación no han funcionado, $y$ genera una respuesta por parte de la autoridad de atención a las demandas o de uso de medidas represivas. Durante el gobierno de Toledo surgieron múltiples espacios de este tipo que demandaban el cumplimiento de promesas electorales (la gran mayoría).

El último campo (el V) tiene características similares al campo III ya que es diálogo con autoridades pero tiene su origen en el campo II, es decir, en la participación conflictiva. Este campo debe entenderse secuencialmente: la protesta origina que la autoridad tome decisiones en el momento (sobre todo cuando la violencia es aguda) pero también que abra espacios hechos a medida de la situación (espacios adhoc) cuando el problema es complejo y demanda una gestión especializadas desde los sectores del Estado involucrados. En estos espacios pueden participar representantes del Poder Ejecutivo de diferentes carteras (Ambiente, Energía y Minas, Salud, Educación, etc.) considerando las demandas y necesidades de los actores primarios. Sin embargo, como veremos más adelante, esta multiplicidad de sectores, y la interseccionalidad de los temas, complejizan el abordaje del espacio de diálogo desde el Estado.

\section{Desempeño del Estado en la gestión de los conflictos sociales y en los espacios de diálogo}

Existen diversos enfoques para abordar la gestión de los conflictos sociales. En general, se considerará en este artículo la propuesta de Cordula Reinmann basada en tres vías: solución, resolución y transformación (Reimann, 2000, pp. 3-7). Las primeras dos vías han sido las formas tradicionales de abordar el conflicto, y corresponden a estrategias de regulación de conflictos (primera vía) y estrategias de resolución de conflictos (segunda vía). La tercera vía es un enfoque de transformación de las condiciones tanto de las que originan el conflicto como de las resultantes.

En esta luz, se ha sometido a análisis diez casos representativos ${ }^{12}$ y se llegó a la conclusión de que cada caso ha tenido diversos tipos de intervención que han sido motivados por la urgencia ante medidas de protesta más o menos violentas. Otro motivador de la intervención más articulada del Estado ha sido la capacidad de articulación de los actores demandantes para movilizarse o para llevar sus reclamos de agendas particulares (en dos sentidos: territorial y temático) hacia temas regionales o de desarrollo.

Por otro lado, los espacios de diálogo se forman por pedido de alguna de las partes, que no siempre es el Estado. De acuerdo con información de la Defensoría del Pueblo (Defensoría del Pueblo, 2017, pp. 51-52), el $21 \%$ de los espacios de diálogo conformados fueron a petición de los actores de la sociedad civil, y el $14 \%$ a petición de las empresas, y el restante $65 \%$ se iniciaron por pedido de alguna instancia del Estado (del gobierno central, regional o local, o de organismos autónomos). Este dato nos da una idea de la proporción de la iniciativa al diálogo y de que esta no es un patrimonio de un tipo de actor ${ }^{13}$.

Ahora, que el Estado tenga la mayor proporción de casos en los que tomaron la iniciativa de entablar el diálogo no quiere decir que su primera respuesta haya sido dialogar. En las etapas más insipientes de los conflictos siempre hay un acercamiento por parte de los actores hacia su contraparte para exponer el

12 Sechura-Americas Potash (Piura), Cuatro Cuencas (Loreto), Mercado Modelo de Piura, Ley Pulpín (Nacional), Quellaveco (Moquegua), Huachón - Statkraft (Pasco), Ocuviri - Ciemsa (Puno), Ocuviri Arasi (Puno), Natucultura (Piura), Cayaltí (Lambayeque).

13 En adelante, los datos a los que se hace referencia son todos de Defensoría del Pueblo. 
Cuadro 3

Resumen de enfoques de gestión en los casos analizados

\begin{tabular}{|c|c|c|c|c|}
\hline \multirow{2}{*}{ Caso } & \multirow{2}{*}{ Ubicación } & \multicolumn{3}{|c|}{ Enfoques de la gestión de conflictos } \\
\hline & & Solución & Resolución & Transformación \\
\hline Sechura-Americas Potash & Piura & $\mathrm{X}$ & $\mathrm{X}$ & \\
\hline Cuatro Cuencas & Loreto & & & $\mathrm{X}$ \\
\hline Mercado Modelo de Piura & Piura & $\mathrm{X}$ & & \\
\hline Ley Pulpín* & Nacional & $\mathrm{X}$ & & \\
\hline Quellaveco & Moquegua & & $\mathrm{X}$ & \\
\hline Huachón-Statkraft & Pasco & & $\mathrm{X}$ & \\
\hline Ocuviri-Ciemsa & Puno & $\mathrm{X}$ & $\mathrm{X}$ & \\
\hline Ocuviri-Arasi & Puno & $\mathrm{X}$ & $\mathrm{X}$ & $\mathrm{X}$ \\
\hline Natucultura & Piura & $\mathrm{X}$ & $\mathrm{X}$ & \\
\hline Cayaltí & Lambayeque & $\mathrm{X}$ & & \\
\hline
\end{tabular}

problema y buscar alternativas de solución. La falta de respuesta o, en menor medida cuando la respuesta no es satisfactoria, genera la intensificación del proceso. La posición del Estado de plantear la conformación de un espacio de diálogo está condicionada al surgimiento de hechos de violencia. A diciembre de 2016, el 53,1\% de casos pasaron a etapa de diálogo después de un hecho de violencia.

Los conflictos en etapa de diálogo son la mitad, ${ }^{14}$ por lo que estamos hablando de una cuarta parte del total de casos que logran ser atendidos en mesas de diálogo a partir del uso de la fuerza. Este dato, sin duda, no es una estadística que sea de conocimiento pleno de los actores que se encuentran en una fase temprana del conflicto, pero hay que tener en cuenta que los medios de comunicación masiva solo se enfocan en los casos a partir de los hechos de violencia. La sensación en la opinión pública (o al menos en la opinión publicada) es que el Estado llega únicamente cuando la violencia ha desbordado. Una búsqueda rápida en la sección de noticias de Google nos arroja resultados claros: los conflictos en los medios de comunicación son lo mismo que las protestas sociales.

La consecuencia de esta visión (real o aumentada) es que según un estudio de opinión ${ }^{15}$, más del $70 \%$ de los peruanos están de acuerdo o muy de acuerdo con

14 Entre 2013 y 2015 eran el $49,1 \%$ y a diciembre de 2016 eran el $51,9 \%$.

15 Encuesta elaborada por el Instituto de Opinión Pública de la Pontificia Universidad Católica del Perú a solicitud de la Defensoría del Pueblo, entre el 12 y 20 de octubre del 2013 sobre una muestra de 1203 personas. que las movilizaciones y protestas sociales son la única forma de ser escuchados por las autoridades. Queda bastante claro que la protesta violenta tiene ganado un prestigio como el mejor camino de llegar al diálogo.

La conformación de los espacios de diálogo, sobre todo si son estructurados, toman un tiempo considerable. De los 84 casos registrados por la Defensoría del Pueblo entre 2013 y 2015, casi el 60\% tomaron hasta un mes en iniciar el diálogo luego de haber sido expuesta públicamente la demanda o de conocido el problema. La cuarta parte tomaron entre 1 y 3 meses, y el resto de 4 meses a más de un año. En este panorama un mes puede parecer poco tiempo, pero si consideramos que casos como Natucultura en Piura se desbordaron en menos de 3 días, un mes puede ser demasiado tiempo.

\section{Cuadro 4}

Tiempo transcurrido entre el inicio del conflicto social y

la conformación del espacio de diálogo, 2013-2015

\begin{tabular}{|l|c|c|}
\hline Tiempo & Total (en meses) & $\%$ \\
\hline Total & 83 & 100,0 \\
\hline Hasta un mes & 49 & 59,0 \\
\hline Entre 1 y 3 meses & 20 & 24,1 \\
\hline Entre 4 meses y un ańo & 9 & 10,8 \\
\hline Más de un ańo & 5 & 6,0 \\
\hline
\end{tabular}

Fuente. Defensoría del Pueblo, 2017, pág. 45.

Otro dato a considerar (Defensoría del Pueblo, 2017, p. 56), es que cuando se compara entre espacios de diálogo estructurados y no estructurados, la proba- 
bilidad de éxito es marcadamente distinta. Un espacio de diálogo estructurado es aquel en el cual se establece claramente el mecanismo de diálogo, se acredita a los participantes, se establecen reglas y roles, y se conoce la frecuencia de las reuniones. Los espacios de diálogo estructurados fueron exitosos en el 33,7\% de los casos, mientras que los no estructurados solo fueron $6,7 \%$. La estructuración del espacio es una tarea que recae fundamentalmente en el Estado, no solo por las competencias que tiene sino por la experiencia acumulada de sus oficinas de gestión de conflictos.

El análisis del tiempo transcurrido hasta el inicio del diálogo se hizo en base únicamente a los casos que pasaron a fase de diálogo, ya que se entiende que el diálogo no es la única vía para la gestión. En ese sentido, toca mirar las razones por las cuáles estos procesos tomaron tanto tiempo en devenir en espacios de diálogo. Considerando únicamente los espacios estructurados o parcialmente estructurados, la principal dificultad para el inicio del diálogo fue la identificación de los interlocutores (33,1\% de los casos), tanto de los demandantes, de los demandados como de los mediadores o intermediadores. ${ }^{16}$

Esta dificultad recae básicamente en los representantes del Estado, ya que los actores de la sociedad civil en muy poco casos (menos del 1\%) son población no organizada. Las protestas las llevan a cabo comunidades campesinas, frentes de defensa, gremios, sindicatos, comunidades nativas, rondas campesinas, etc., es decir organizaciones con estructura y roles orgánicos definidos. Por otro lado, las empresas — considerando que son organizaciones jerarquizadas - tienen bien definidos a sus representantes (los equipos de relaciones comunitarias o los gerentes en última instancia).

Sorteando la dificultad de la identificación de los interlocutores (incluidos los del Estado, quienes muchas veces no tienen las competencias suficientes para desempeñar este rol), es importante no perder de vista que los procesos de conflictos son un asunto público en tanto bienes jurídicos pueden verse comprometidos. La dinámica del proceso puede involucrar (y lo ha hecho en muchos casos) que la protesta continúe durante el diálogo, y cuando esta se presenta, la respuesta del Estado ha sido la declaratoria del estado de emergencia o el envío policías o de fuerzas militares.

16 Otras dificultades fueron la elección del lugar donde desarrollar las reuniones $(29,2 \%)$, la dinámica misma de las reuniones $(31,8 \%)$, la conducción inadecuada del proceso $(8,9 \%)$, entre otras.
La estrategia de respuesta del Estado siempre ha sido distinta pero la cifra de personas heridas y/o fallecidas nos da luces para pensar que las intervenciones policiales (o militares) no son la mejor opción para cautelar los derechos humanos ni otros bienes jurídicos que el estado debe proteger.

\section{El uso de la violencia como medio}

Durante la fase temprana de un caso, los actores hacen públicas sus demandas, necesidades y posiciones, lo que significa que ya han agotado los intentos de canalizar el proceso en los espacios (institucionalizados o no) de deliberación. Este hecho ya genera en el actor demandante una acumulación de tensión que se va plasmando en la posición que tengan frente a su contraparte. Si a esto le sumamos la indiferencia del Estado para mediar o responder ante sus demandas, o el desprecio o falta de reconocimiento a sus dirigentes o representantes, o una mirada culturalmente hegemónica sobre sus necesidades, o una respuesta no comprensiva y desproporcionada por parte de las fuerzas del orden, o incluso el ataque político o ideológico sobre sus perspectiva del problema, estamos frente a un caldo de cultivo para el desencadenamiento de respuestas violentas.

En este panorama, el ingrediente final es el prestigio que a lo largo de los años el uso de la violencia ha ganado como recurso para conseguir la atención de parte del Estado. Líneas arriba se mencionó el alto porcentaje de casos que pasan a etapa de diálogo luego de ocurridos hechos de violencia (más de la mitad).

\section{Algunas conclusiones}

A lo largo de este artículo se han planteado tres ideas fundamentales:

i. El Estado peruano no cumple con el papel de interlocutor en los conflictos sociales.

ii. La falta de un interlocutor en un conflicto social, impide el surgimiento de espacios de diálogo.

iii. La falta de espacios de diálogo para la resolución de conflictos producen la intensificación de la protesta que puede conllevar al uso de la violencia 


\section{Discusión final}

Respecto de la primera idea, se ha descrito que existen diversas estrategias de gestión de los conflictos sociales que parten desde qué entienden los funcionarios del Estado sobre lo que es un conflicto. La perspectiva más común en los casos en estudio (y en general en los diversos conflictos que surgieron en el período en estudio) es del conflicto como un problema del status quo y del orden social, lo que requiere una estrategia de solución que va por el lado de las sanciones, la mediación imperativa, la imposición de la paz y en otros casos la negociación no coercitiva.

Estamos pues ante un Estado que no cumple un rol de interlocutor ya que considera el conflicto como un problema en sí mismo y no como el síntoma de un problema mayor que requiere del concurso de diversos actores de la sociedad y el Estado para darle una salida consensuada. Pero más importante aún, estamos ante un Estado peruano que no tiene la capacidad real para representar un rol coherente frente a los conflictos y menos ante un espacio de diálogo. Esto pasa por una inadecuada (y muy cambiante) organización de su aparato y funcionarios, y la poca capacitación, experiencia y recursos de los que disponen para asumir sus funciones. En ese sentido, lo que hemos visto en los casos analizados y en la estadística, es que cada caso tiene al frente a un Estado diferente, con una actitud distinta, con una estrategia ad-hoc, con una capacidad de negociar asimétrica y con resultados dispares. Podemos asegurar, firmemente, que el Estado peruano no cumple con su rol de interlocutor.

Respecto a la segunda idea, se ha visto que existen diversos campos de participación, institucionalizados y no institucionalizados, de imposición de la voluntad ciudadana y de diálogo con las autoridades (permanentes y ad-hoc). Estos canales de participación tienen características distintas y usos diversos, siendo el más recurrente en los casos estudiados (y en los conflictos sociales en general) el de la protesta y los movimientos sociales.

Los mecanismos que se encuentran en este campo son requeridos por las organizaciones sociales y los actores de la sociedad civil en general porque son más efectivos, menos costosos, y pueden ser la entrada a otros mecanismos de diálogo ad-hoc con las autoridades que no se generarían sin la protesta previa. Tenemos entonces que el Estado es constantemente forzado a sentarse a dialogar o a ejercer el rol de mediador o facilitador del conflicto social.

Los actores sociales que participan en conflictos sociales no hacen uso de los canales de participación por los motivos ya explicados, con lo que despliegan sus acciones en el campo de la protesta como una forma de conseguir un espacio ad-hoc (mesa de diálogo, mesa de trabajo, comité de gestión, etc.) que les facilite la toma de acuerdos. Sin embargo, el desempeño de los funcionarios y/o servidores públicos es heterogéneo y lento, haciendo que las decisiones se tomen inoportunamente, o que los acuerdos no se lleguen a cumplir. En síntesis, el Estado depende de la capacidad de decisión que pueda tener el funcionario principal a cargo de dirigir el diálogo. Cada espacio conformado tiene un inicio más o menos similar pero no existe certeza de cómo va a terminar.

Finalmente, respecto a la tercera idea, se mostró que la violencia tiene un prestigio ganado en la protesta social ya que al menos la mitad de los espacios de diálogo que surgieron en el período en estudio se abrieron luego de hechos de violencia social. Hemos visto también que esta violencia no solo genera restricción en el tránsito o el cese de actividades económicas, sino que deja un legado de personas heridas y fallecidas.Las consecuencias de la violencia son la vulneración de derechos (de todas las generaciones) pero también el surgimiento y desarrollo de un sentido común que empuja a actores sociales y al Estado a relacionarse y desenvolverse bajo la amenaza constante del surgimiento de la violencia.

Este sentido común convive, de hecho, con la cultura del diálogo que existe en el país. Las bases culturales de la mayoría de peruanos son orales, no escritas, razón por la cual la palabra dicha puede llegar a tener un peso mucho mayor que la palabra escrita. De hecho, muchos procesos de diálogo se caen no porque los acuerdos escritos no reflejen la atención de las necesidades del actor protestantes, sino porque en el diálogo se dijeron cosas que pusieron en duda la credibilidad del firmante. Muchos funcionarios terminan siendo vetados por cosas que han dicho.

El pedido cada vez más recurrente de la presencia de altos funcionarios en los espacios de diálogo (ministros, primer ministro, presidente) responde a que la credibilidad de los técnicos ha mermado y las mismas actas de acuerdos pierden valor ante el incumplimiento constante. Es un escenario en el que se 
busca escuchar de la misma boca del funcionario que va a atender la problemática subyacente al conflicto. Podemos decir claramente que mientras que la violencia ha ganado prestigio, las actas de acuerdos han ido perdiéndolo.

Dicho sea de paso, las heridas de la violencia en el tejido social son las más difíciles de tratar y de hecho el Estado no ha llevado a cabo hasta el momento ningún esfuerzo de gestionar el post conflicto, en el sentido de restituir las relaciones Estado-sociedad y mucho menos ha hecho un reconocimiento de que la violencia ha sido en muchos casos el producto de una atención inadecuada del conflicto social (siendo uno de los casos más dramáticos el caso de Bagua).

Podemos concluir entonces que existe una relación entre la incapacidad del Estado para atender los conflictos sociales oportunamente y por la vía de la transformación, y el escalamiento de la violencia en la protesta social y la profundización de los problemas estructurales que sirven de generadores del conflicto social. Estamos ante un círculo vicioso de violencia y falta de atención de la misma.

\section{Bibliografía}

Arellano Yanguas, J. (2014). ¿Minería sin fronteras? Conflicto y desarrollo en regiones mineras del Perú. Lima: IEP.

BAquer, M. A. (2000). Ralf Darehndorf: una cierta idea, entre liberal y socialdemócrata, de la realidad europea. Cuadernos de estrategia (N. $\left.{ }^{\circ} 105\right)$, pp. 154-182.

Bedoya, C., Caravedo, J., Moreno, G., Ormachea, J., \& Yeomans, C. (2007). Manejo constructivo de conflictos y promoción de consensos. Lima: ProDiálogo-Prevención y Resolución de Conflictos.

Bourdieu, P. (1999). Razones prácticas. Sobre la teoría de la acción. Barcelona: Anagrama.

Caravedo, J., \& Moreno, G. (2007). Construcción y fortalecimiento de espacios de diálogo. Lima: ProDiálogoPrevención y Resolución de Conflictos.

Coser, L. (1961). Las funciones del conflicto social. México D.F.: Fondo de Cultura Económica.

De Echave, J. (2001). En J. De Echave, Construyendo un proceso de toma de decisiones frente a operaciones mineras. Lima: CooperAcción-Acción Solidaria para el Desarrollo.
De Echave, J., Keenan, K., Romero, M. K., \& Tapia, Á. (2005). Los procesos de Diálogo y la Administración de Conflictos en Territorios de Comunidades: El Caso de la Mina de Tintaya en el Perú. Lima: CooperAccion.

Defensoría Del Pueblo (2012). Violencia en conflitos sociales. Lima: Defensoría del Pueblo.

Defensoría del Pueblo (2017). El valor del diálogo. Lima: Defensoría del Pueblo.

Entelman, R. F. (2002). Teoría de conflictos: hacia un nuevo paradigma. Barcelona: Gedisa.

Galtung, J. (1996). Peace by peaceful means. Peace and conflict, development and civilization. Londres: Sage.

Grompone, R., \& TANaKa, M. (2009). Entre el crecimiento económico y la insatisfacción social: las protestas sociales en el Perú actual. Lima: IEP.

Gruppi, L. (1978). El concepto de hegemonia en Gramsci. México D.F.: Ediciones de Cultura Popular.

Lenin, V. I. (1974). ¿Qué hacer? Pekín: Ediciones en Lenguas Extranjeras.

Lorenzo Cadarso, P. L. (1995). Fundamentos teóricos del conflicto social. Norba (N. $\left.{ }^{\circ} 15\right)$. Revista de Historia., págs. 237-253.

Marx, K., \& Engels, F. (2011). Manifiesto del Partido Comunista. México D.F.: Centro de Estudios socialistas Carlos Marx.

Olson, M. (1985). Auge y decadencia de las naciones. Barcelona: Ariel.

Pruitt, D. G., \& Kim, S. H. (2004). Social Conflict: Escalation, Stalemate and Settlement. Nueva York: McGraw-Hill.

REIMANN, C. (2000). Hacia la resolución de conflictos: evaluación de los enfoques recientes de gestión de conflictos. En C. Reimann, The Berghof Handbook for Conflict Transformation. Berlín: Berghof Research Center for Constructive Conflict Management.

Remy, M. I. (2005). Los múltiples campos de la participación ciudadana en el Perú: un reconocimiento del terreno y algunas reflexiones. Lima: IEP.

Tilly, C. (1978). From Mobilization to Revolution. Nueva York: Random House-McGraw-Hill.

Vinyamata, E. (2014). Conflictología. Barcelona: Ariel.

World Health Organization - WHO. (15 de enero de 2019). Violence Prevention Alliance. Obtenido de https://www.who.int/violenceprevention/approach/ definition/en/ 